\title{
A low-frequency radio halo associated with a cluster of galaxies
}

G. Brunetti ${ }^{1}$, S. Giacintucci ${ }^{1,2}$, R. Cassano ${ }^{1}$, W. Lane $^{3}$,

D. Dallacasa ${ }^{4}$, T. Venturi ${ }^{1}$, N. Kassim ${ }^{3}$, G. Setti ${ }^{1,4}$,

W. D. Cotton ${ }^{5}$, \& M. Markevitch ${ }^{2}$

${ }^{1}$ INAF - Istituto di Radioastronomia, Via P. Gobetti 101, I-40129 Bologna, Italy

${ }^{2}$ Harvard-Smithsonian Center for Astrophysics, Cambridge, MA 02138, USA

${ }^{3}$ Naval Research Laboratory, Code 7213, Washington, DC 20375-5320, USA

${ }^{4}$ Dipartimento di Astronomia, Universita' di Bologna, Via Ranzani 1, I-40127 Bologna, Italy

${ }^{5}$ National Radio Astronomy Observatory, Charlottesville, Virginia 22903-2475, USA

Clusters of galaxies are the largest gravitationally bound objects in the Universe, containing about $10^{15}$ solar masses of hot $\left(10^{8} \mathbf{K}\right)$ gas, galaxies and dark matter in a typical volume of about $10 \mathrm{Mpc}^{3}$. Magnetic fields and relativistic particles are mixed with the gas as revealed by giant radio haloes, which arise from diffuse, megaparsec-scale synchrotron radiation at cluster center ${ }^{1,2}$. Radio haloes require that the emitting electrons are accelerated in situ (by turbulence) ${ }^{3-6}$, or are injected (as secondary particles) by proton collisions into the intergalactic medium ${ }^{7-10}$. They are found only in a fraction of massive clusters that have complex dynamics ${ }^{11-14}$, which suggests a connection between these mechanisms and cluster mergers. Here we report a radio halo at low frequencies associated with the merging cluster Abell521. This halo has an extremely steep radio spectrum, which implies a high frequency cut-off; this makes the halo difficult to detect with observations at $1.4 \mathrm{GHz}$ (the frequency at which all other known radio haloes have been best studied). The spectrum of the halo is inconsistent with a secondary origin of the relativistic electrons, but instead supports turbulent acceleration, which suggests that many radio haloes in the Universe should emit mainly at low frequencies.

The turbulent re-acceleration mode ${ }^{5,6,15-17}$ for the origin of giant radio haloes assumes 
that fossil relativistic particles are re-accelerated by merger-induced turbulence to the energies necessary to produce the observed radio synchrotron emission in relatively weak magnetic fields. The acceleration of fast particles by turbulence, known to be an important process in astrophysics, is due to the resonant scattering of these particles by the turbulent waves (the energy spectrum of the turbulence can be thought of as the superposition of the contribution from turbulent waves with different length scales), leading to a stochastic energization of particles and to the damping of the waves ${ }^{18}$. The connection between mergers and particle acceleration by turbulence in galaxy clusters is complex. It is argued $15,19,20$ that it should take place on a timescale of the order of a clustersubcluster crossing time ( $\sim 1 \mathrm{Gyr})$, during which turbulence is continuously injected on scales of the order of the subcluster size, transported at smaller scales and then dissipated into heating of the intergalactic medium and acceleration of relativistic particles over a fairly large volume. This argument is supported by recent radio observations of a complete sample of X-ray luminous clusters that allow a clear separation between clusters with radio haloes and radio-quiet clusters, suggesting, from the fraction of clusters with radio haloes, that the particle acceleration mechanisms operate sporadically, on timescales $\leq 1 \mathrm{Gyr}$ and in connection with cluster mergers ${ }^{21}$. Abell521 is an X-ray luminous $\left(8.2 \times 10^{37} \mathrm{~W}\right.$ in the $0.12 .4-\mathrm{keV}$ band $)$ and massive $\left(\sim 2 \times 10^{15}\right.$ solar masses $)$ galaxy cluster at redshift $\mathrm{z}=0.247$ with ongoing multiple merging episodes ${ }^{22,23}$. Here we report the discovery of a giant radio halo in this cluster by means of deep observations with the Giant Metrewave Radio Telescope (GMRT, India) at 240, 325 and 610MHz. In Fig. 1a we show the radio image of Abell521 at $240 \mathrm{MHz}$, where the radio halo is best imaged. To highlight the diffuse emission, point sources visible in the full-resolution images were subtracted in producing the low-resolution images in Fig. 1. The radio halo is coincident with the cluster X-ray emitting region and correlates with the X-ray emission of the hosting cluster, which is typical of 
other radio haloes $^{1,2}$ (see also Supplementary Information). Previous higher frequency Very Large Array (VLA) observations at $1,400 \mathrm{MHz}$ did not find this diffuse emission, instead observing only the radio relic located on the southeastern boundary of the cluster ${ }^{23}$. The relic coincides with a possible shock front, generated by recent in-fall of a subcluster along the northwest/southeast direction, where relativistic electrons are currently accelerated ${ }^{24}$. Figure 1 clearly shows that the radio halo becomes progressively more dominant over the radio relic at lower frequencies, indicating that its spectrum is much steeper than that of the relic, which has $\alpha \approx 1.5$ (ref. 24; flux is proportional to $v^{-\alpha}$, where $v$ denotes frequency). The patchy structure of the radio halo at $610 \mathrm{MHz}$ (Fig. 1b) indicates the observational difficulty in imaging the emission, the surface brightness of which is already fading at this frequency (note that the largest diffuse emission detectable with the GMRT at $610 \mathrm{MHz}$ is $\approx 0.28$ degrees, e.g. ref. 14). The halo disappears between 610 and 1,400MHz; only an upper limit on the flux of the radio halo at $1,400 \mathrm{MHz}$ can be derived, although faint residual emission in the cluster is still present at this frequency (Fig. 1c and Supplementary Information). The flux densities of the radio halo at 240,325 and $610 \mathrm{MHz}$ are plotted in Fig. 2 together with the upper limits at 74 and $1,400 \mathrm{MHz}$. The upper limits were evaluated by injecting fake radio haloes with different flux densities into the observed data sets, following ref. 21 , to estimate the sensitivity of the observations to diffuse emission on the halo length scale. In particular, the upper limit at $74 \mathrm{MHz}$ was derived from VLA Low-frequency Sky Survey data ${ }^{25}$, whereas the upper limit at $1,400 \mathrm{MHz}$ was derived from the analysis of the archival VLA data (Supplementary Information). The important result is that the average value of the spectral index, $\alpha \approx 2.1$, is much larger than that of any other known radio halo (typical spectral index is $\alpha \approx 1.2-1.3$ (refs 1,2)). These extreme spectral properties make Abell521 a unique system for addressing the origin of the emitting particles in radio haloes. Such an extremely steep spectrum and 
the downward spectral curvature (Fig. 2) imply a spectral cut-off at high frequency, which is a well-known signature of turbulent acceleration ${ }^{4-6,16,17,19}$. Synchrotron theory implies a corresponding cut-off in the spectrum of the emitting electrons at $E_{e} \approx 1.4 B_{n T}^{-1 / 2}\left(v_{c} / 300\right)^{1 / 2}$ $\mathrm{GeV}$, where $v_{c}$ is the cut-off frequency (measured in megahertz) and $B_{n T}$ is the magnetic field (measured in $\mathrm{nT}$ ). In the framework of the turbulent acceleration scenario, $E_{e}$ pinpoints the energy at which the timescale of the electron radiative losses becomes equal to that of the turbulent acceleration. The timescale of electrons emitting at $v_{c}$ is estimated taking into account the redshift-dependent inverse-Compton losses against the cosmic microwave background and the synchrotron $\operatorname{losses}^{26}$, as follows, where $v_{c}$ is again measured in megahertz:

$$
\tau \approx 0.95 \frac{B_{n T}^{1 / 2}\left(v_{c} / 300\right)^{-1 / 2}}{(1+z)^{4}+\left(B_{n T} / 0.32\right)^{2}} \mathrm{Gyr}
$$

This means that the electrons responsible for the observed emission should be accelerated on a timescale of $\sim(1.1-1.4) \times 10^{8}$ years, for $0.1-0.5$-nT magnetic fields in the radio halo region. Assuming that fast magnetosonic waves are responsible for the acceleration of the emitting particles, following ref. 20 we find that this acceleration efficiency can be achieved under the reasonable assumption that the energy density of these turbulent waves is $12-18 \%$ of the thermal energy (Fig. 2).

Appreciable synchrotron emission can also be produced by secondary electrons injected by collisions between long-lived relativistic protons accumulated in the cluster and the thermal protons in the intergalactic medium, and secondary models have been proposed as alternatives to the re-acceleration model to explain radio haloes ${ }^{7-10}$. The very steep spectral slope of this radio halo rules out secondary models by means of a straightforward energy argument. To explain the spectrum of the radio halo through synchrotron radiation from secondary electrons, 
the primary protons must have a very steep spectral energy distribution $\left(N(p) \propto p^{-\delta}\right.$, where $\delta \approx 4.2$ and $p$ denotes the particle momentum). The energy density of relativistic protons, $\varepsilon_{p}$, required to match the observed synchrotron flux through a secondary model can be estimated following the formalism in ref. 27. For an average number density of thermal protons of $n_{t h} \approx 1,500 \mathrm{~m}^{-3}$ in the region of the radio halo (consistent with the average thermal density in the same region derived from X-ray observations ${ }^{22}$ ) and the synchrotron flux measured at $325 \mathrm{MHz}$, we find that $\varepsilon_{p}$ ranges from approximately 3 to 100 times the energy density of the thermal plasma for magnetic field values (averaged in the region of the radio halo) ranging from $B=0.5 n T$ to $B=0.1 \mathrm{nT}$, respectively. This yields only a lower limit on the energy density of high-energy protons, because for $\delta>3$ an additional (even dominant) contribution to the energy comes from suprathermal particles with kinetic energies $<1 \mathrm{GeV}$. A secondary origin for the emitting electrons thus implies the unrealistic situation in which clusters are dominated by non-thermal protons. It also violates present upper limits on the energy density of these particles derived from $\gamma$-ray observations of several clusters, at the $20 \%$ level $^{28,29}$. If the spectrum of these protons has $\delta<4$, then the synchrotron signal from the secondary electrons produced by proton-proton collisions cannot exceed the 1.4-GHz upper limit in Fig. 2, placing corresponding limits on the energy density of the primary protons. Figure 3 shows upper limits on the energy density of the primary protons in the region of the radio halo as a function of the magnetic field. These limits were obtained following ref. 21 and show that the energy density of relativistic protons in the cluster is less than $1 \%$ of the thermal component for $B>0.2 \mathrm{nT}$ and $\delta<2.5$. A similar conclusion has recently been reached in the analysis of a statistical sample of galaxy clusters without radio haloes ${ }^{21}$. However, in that case a larger energy content of protons was still possible by assuming that clusters without radio haloes have magnetic fields much smaller than those with radio haloes. This alternative (ad hoc) 
possibility can be reasonably ruled out in our case because Abell521 hosts a radio halo with bolometric radio luminosity comparable to that of classical radio haloes $\left(v P(v) \approx 10^{34} \mathrm{~W}\right)$. Future observations with the Fermi Gamma-ray Space Telescope (formerly the Gamma-ray Large Area Space Telescope) will reveal galaxy clusters in $\gamma$-rays in cases where the energy content of relativistic protons is significantly larger than about $1 \%$ of the thermal plasma. The combination of this future data and limits in the radio band (Fig. 3 and ref. 21) may thus provide a powerful tool for constraining the magnetic field strength in galaxy clusters.

As we look at applying the turbulent re-acceleration model to other galaxy clusters, it should be stressed that the maximum energy to which electrons can be re-accelerated and, ultimately, the cut-off frequency in the spectra of radio haloes depend on the level of turbulence and on the properties of the turbulent waves. The spectral cut-off affects our ability to detect radio haloes in the Universe, introducing a strong bias against observing them at frequencies substantially larger than $v_{c}$. Currently known radio haloes are observed at gigahertz frequencies, requiring an efficient turbulent acceleration mechanism. These haloes must result from the rare, most energetic merging events and are therefore hosted only in the most massive, hottest clusters ${ }^{19,30}$, in line with observations ${ }^{11-14}$. On the other hand, the majority of radio haloes should form during much more common, but less energetic, merging events, for example between a massive cluster and a substantially smaller subcluster (with mass ratio $>5$ ) or between two similar clusters with mass $\leq 10^{15}$ solar masses $^{13,30}$. However, these sources, with a cut-off in the synchrotron spectrum at $v_{c}<1 \mathrm{GHz}$, should be visible only at lower frequencies, because their spectrum should be similar to that of the low-frequency radio halo in Abell521. Future high-sensitivity radio telescopes operating at low frequencies, such as the Low Frequency Array (LOFAR) and the Long Wavelength Array (LWA), are expected to discover the majority of these sources and also to test their connection with cluster mergers. 

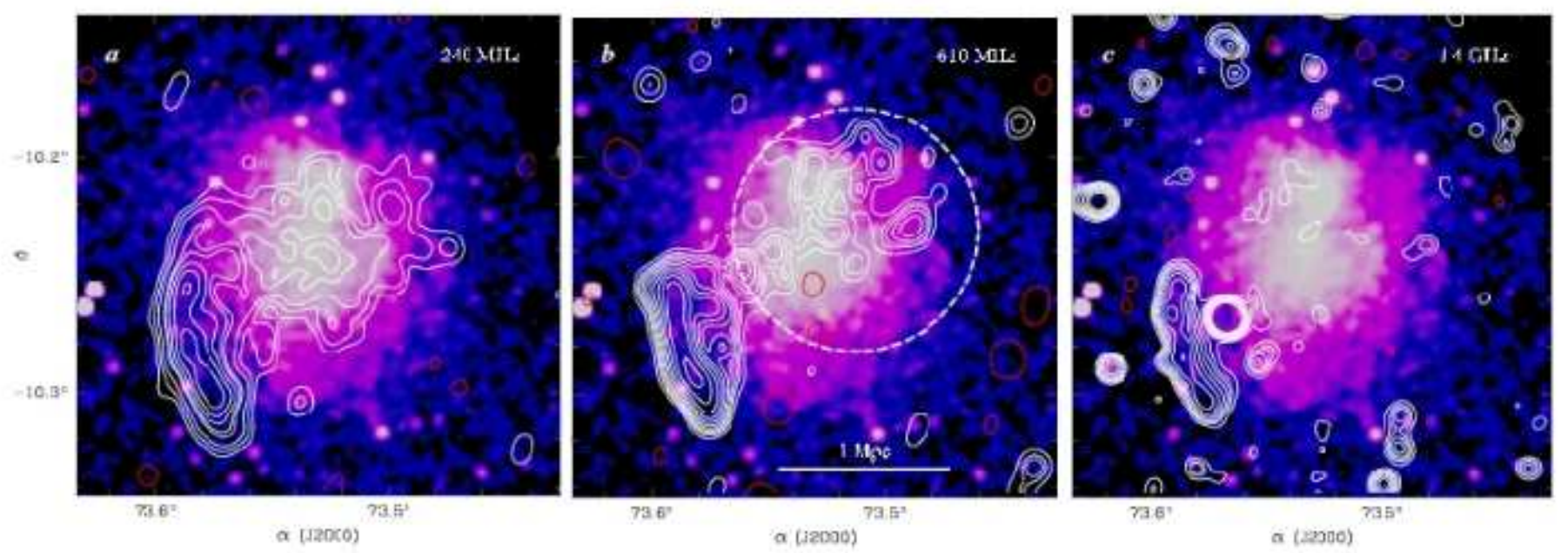

Figure 1: Radio and X-ray images of Abell 521. Low resolution radio contours (after subtraction of discrete sources in the field identified at full resolution) overlaid on the Chandra $0.5-4 \mathrm{keV} \mathrm{X}$-ray image (corrected for background and exposure and smoothed with a $\sigma=6$ arcsec Gaussian, as in ref. 24). Panel a): GMRT $240 \mathrm{MHz}$ contours at $35 \operatorname{arcsec} \times 35 \operatorname{arcsec}$ resolution. The r.m.s. noise level in the cluster region is $220 \mu \mathrm{Jy} / \mathrm{beam}$ (full resolution used for subtraction of discrete sources has 15.6 arcsec x $12.3 \mathrm{arcsec}$ beam and r.m.s. noise $=190 \mu \mathrm{Jy} /$ beam). Contours are at -0.66 (red), 0.66, 1.3, 2.6, 5.2, 7, 10, 14, $19 \mathrm{mJy} /$ beam. Panel b): GMRT $610 \mathrm{MHz}$ contours at $35 \operatorname{arcsec} \mathrm{x} 35 \operatorname{arcsec}$ resolution. The r.m.s. noise level in the cluster region is $58 \mu \mathrm{Jy} /$ beam (full resolution used for subtraction of discrete sources has $9 \operatorname{arcsec} \times 4$ arcsec beam and r.m.s. noise $=35 \mu \mathrm{Jy} / \mathrm{beam})$. Contours are at -0.17 (red), 0.17 , $0.34,0.48,0.67,0.94,1.3,1.9,2.6,3.7,5.2 \mathrm{mJy} / \mathrm{beam}$. The dashed circle indicates the region in which fluxes of the radio halo at different frequencies (reported in Fig. 2) are measured. Panel c): VLA 1.4 $\mathrm{GHz}$ contours at $25 \operatorname{arcsec} \times 25 \operatorname{arcsec}$ resolution. Here only the discrete sources within 3 arcmin from the cluster centre are subtracted (other images are presented in Supplementary Information together with a discussion on the subtraction of discrete sources at this frequency). The r.m.s. noise level in the cluster region is $26 \mu \mathrm{Jy} / \mathrm{beam}$ (the full resolution used for subtraction of discrete sources has 12.7 $\operatorname{arcsec} \times 6.9$ arcsec beam and r.m.s. noise $=15 \mu \mathrm{Jy} / \mathrm{beam}$ ). Contours are at -0.08 (red), 0.08, 0.16, 0.22, 0.32, 0.45, 0.63, 0.89, 1.25, 1.76, 2.48, 3.5, 4.9 mJy/beam. Coordinate system, J2000.

At the moment this connection cannot be tested, owing to the lack of observations of samples of galaxy clusters at low radio frequencies.

\section{References}

1. Giovannini, G.; Feretti, L., in L.Feretti, I.M.Gioia, G.Giovannini eds., Diffuse radio sources and cluster mergers: radio halos and relics, ASSL 272, 197-227, Kluwer Aca- 


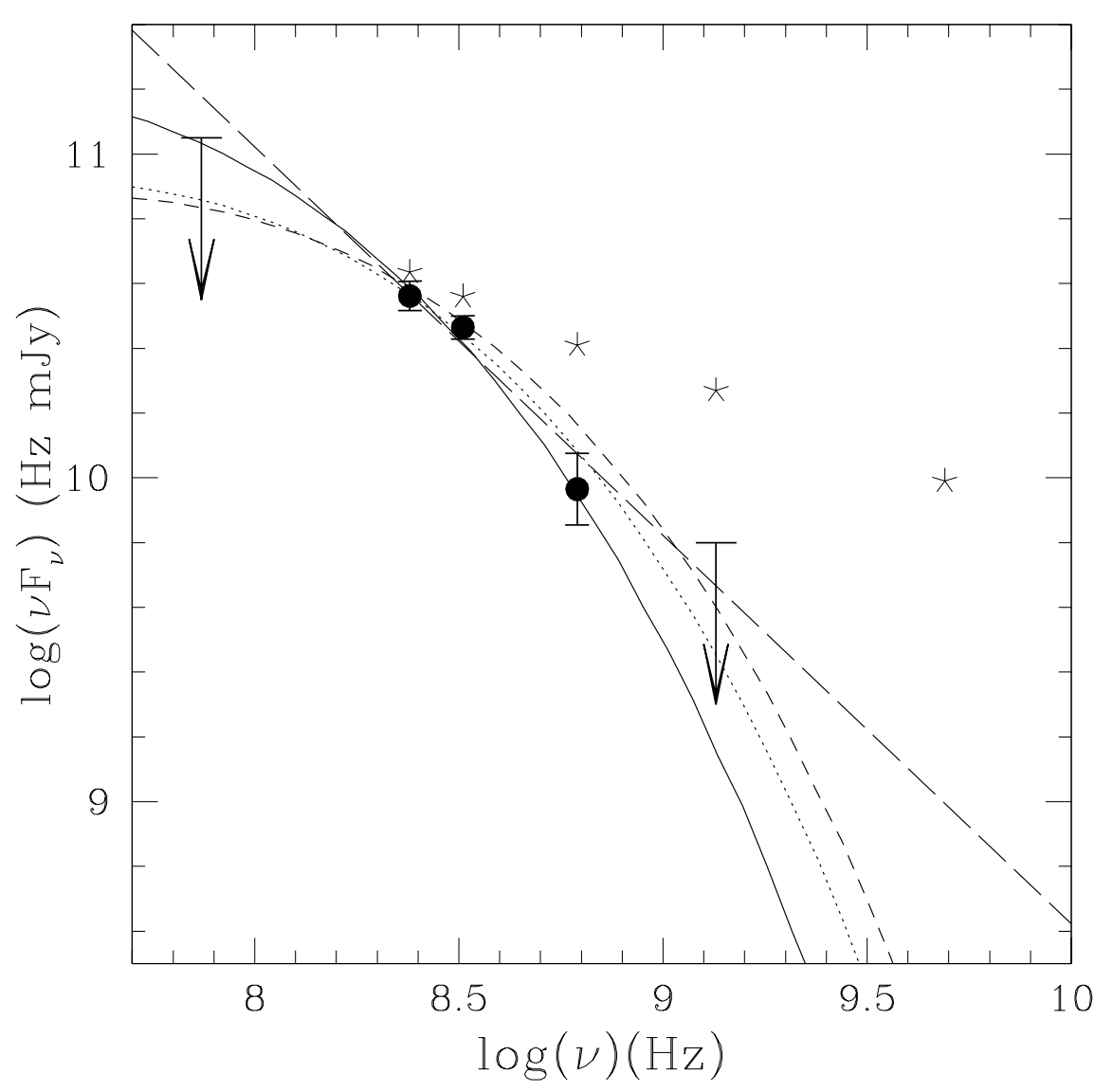

Figure 2: The spectrum of the radio halo. Data are overlaid on secondary (long-dashed line) and re-acceleration models. Measured fluxes $\left(F_{v}\right)$ are : $152 \pm 15 \mathrm{mJy}$ at $240 \mathrm{MHz}, 90 \pm 7 \mathrm{mJy}$ at $325 \mathrm{MHz}$, $15 \pm 3.5 \mathrm{mJy}$ at $610 \mathrm{MHz}$, limits are $1.5 \mathrm{Jy}$ at $74 \mathrm{MHz}$ and $5 \mathrm{mJy}$ at 1,400 MHz; errors (and error bars in the figure) are given at 1 s.d. For comparison, asterisks give the spectral energy distribution of the radio relic (taken from ref.24). The secondary model assumes $\delta=4.2$ and requires that the energy density of relativistic protons is larger than that of the thermal energy (see text). Re-acceleration models assume : $14 \%$ of the thermal energy in magnetosonic waves and a central value of the magnetic field $B_{o}=0.15$ nT (solid curve), $14 \%$ of the thermal energy in magnetosonic waves and $B_{o}=0.35 \mathrm{nT}$ (dotted curve), $18 \%$ of the thermal energy in magnetosonic waves and $B_{o}=0.15 \mathrm{nT}$ (short-dashed curve). All the reacceleration models adopt a scaling $B \propto n_{\text {th }}$ (e.g., ref.2 and refs therein). 


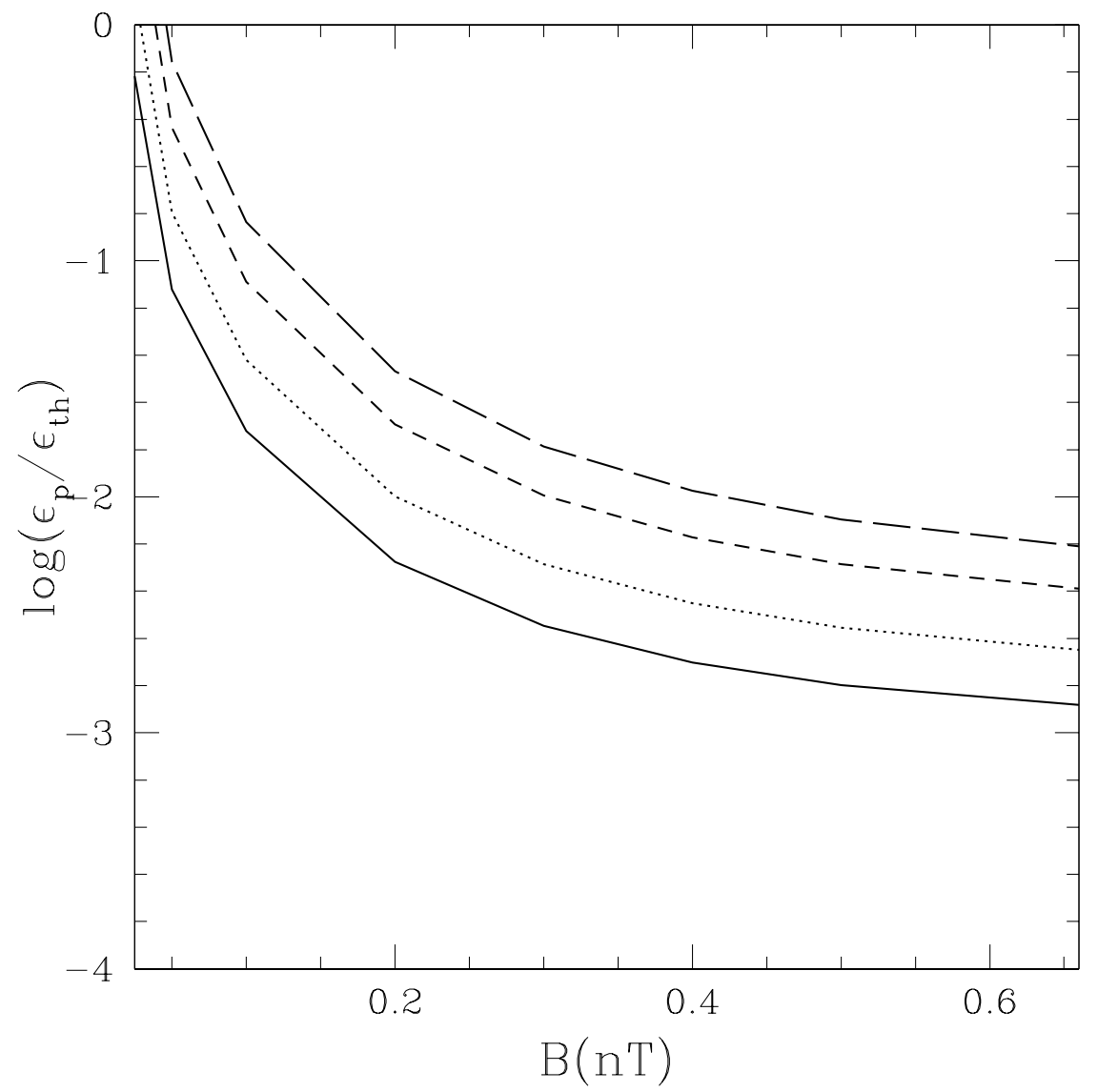

Figure 3: Limits to the energy density of relativistic protons. Curves give the upper limit to the ratio between the energy density of relativistic protons and that of thermal gas, $\varepsilon_{t h}$, as a function of the magnetic field in Abell 521. Calculations are shown assuming $n_{\text {th }}=1,500 \mathrm{~m}^{-3}$ and $k_{B} T=7 \mathrm{keV}$. From bottom to top we adopt a spectra of the primary protons : $\delta=2.3,2.5,2.7,2.9\left(N(p) \propto p^{-\delta}\right)$. 
demic Publishers, Dordrecht (2002)

2. Ferrari, C.; Govoni, F.; Schindler, S.; Bykov, A. M.; Rephaeli, Y., Observations of Extended Radio Emission in Clusters, Sp. Science Rev. 134, 93-118 (2008)

3. Jaffe, W.J., Origin and transport of electrons in the halo radio source in the Coma cluster, Astrophys. J. 212, 1-7 (1977)

4. Schlickeiser, R.; Sievers, A.; Thiemann, H., The diffuse radio emission from the Coma cluster, Astron. Astrophys. 182, 21-35 (1987)

5. Brunetti, G.; Setti, G.; Feretti, L.; Giovannini, G., Particle reacceleration in the Coma cluster: radio properties and hard X-ray emission, Mon. Not. R. Astron. Soc. 320, 365$378(2001)$

6. Petrosian, V., On the Nonthermal Emission and Acceleration of Electrons in Coma and Other Clusters of Galaxies, Astrophys. J. 557, 560-572 (2001)

7. Dennison, B., Formation of radio halos in clusters of galaxies from cosmic-ray protons, Astrophys. J. 239, L93-L96 (1980)

8. Blasi, P.; Colafrancesco, S., Cosmic rays, radio halos and nonthermal X-ray emission in clusters of galaxies, Astroparticle Phys. 12, 169-183 (1999)

9. Dolag, K., Ensslin, T.A., Radio halos of galaxy clusters from hadronic secondary electron injection in realistic magnetic field configurations, Astron. Astrophys. 362, 151-157 (2000)

10. Pfrommer, C.; Ensslin, T.A, Estimating galaxy cluster magnetic fields by the classical and hadronic minimum energy criterion, Mon. Not. R. Astron. Soc. 352, 76-90 (2004) 
11. Giovannini, G.; Tordi, M.; Feretti, L., Radio halo and relic candidates from the NRAO VLA Sky Survey, New Astron. 4, 141-155 (1999)

12. Buote, D.A., On the Origin of Radio Halos in Galaxy Clusters, Astrophys. J. 553, L15L18 (2001)

13. Cassano, R., et al., Revised statistics of radio halos and the reacceleration model, Astron. Astrophys. 480, 687-697 (2008)

14. Venturi, T. et al., GMRT Radio Halo Survey in galaxy clusters at $\mathrm{z}=0.2-0.4$. II.The eBCS clusters and analysis of the complete sample, Astron. Astrophys. 484, 327-340 (2008)

15. Fujita,Y.; Takizawa,M.; Sarazin,C.L., Nonthermal Emissions from Particles Accelerated by Turbulence in Clusters of Galaxies, Astrophys. J. 584, 190-202 (2003)

16. Kuo, Ping-Hung; Hwang, Chorng-Yuan; Ip, Wing-Huen, Diagnostic Signatures of Radio and Hard X-Ray Emission on Particle Acceleration Processes in the Coma Cluster, Astrophys. J. 594, 732-740 (2003)

17. Brunetti, G.; Blasi, P.; Cassano, R.; Gabici, S., Alfvenic reacceleration of relativistic particles in galaxy clusters: MHD waves, leptons and hadrons, Mon. Not. R. Astron. Soc. 350, 1174-1194 (2004)

18. Melrose, D.B., Plasma astrophysics (Gordon \& Breach, New York) (1980)

19. Cassano, R.; Brunetti, G., Cluster mergers and non-thermal phenomena: a statistical magneto-turbulent model, Mon. Not. R. Astron. Soc. 357, 1313-1329 (2005)

20. Brunetti, G.; Lazarian, A., Compressible turbulence in galaxy clusters: physics and stochastic particle re-acceleration, Mon. Not. R. Astron. Soc. 378, 245-275 (2007) 
21. Brunetti, G., et al., Cosmic Rays and Radio Halos in Galaxy Clusters: New Constraints from Radio Observations, Astrophys. J. 670, L5-L8 (2007)

22. Arnaud, M.; Maurogordato, S.; Slezak, E.; Rho, J., A521: A cluster forming at the crossing of two filaments?, Astron. Astrophys. 355, 461-478 (2000)

23. Ferrari, C.; Arnaud, M.; Ettori, S.; Maurogordato, S.; Rho, J., Chandra observation of the multiple merger cluster Abell 521, Astron. Astrophys. 446, 417-428 (2006)

24. Giacintucci, S., et al., Shock acceleration as origin of the radio relic in A521?, Astron. Astrophys. 486, 347-358 (2008)

25. Cohen, A.S., et al., The VLA Low-Frequency Sky Survey, Astron. J. 134, 1245-1262 (2007)

26. Sarazin, C.L., The Energy Spectrum of Primary Cosmic-Ray Electrons in Clusters of Galaxies and Inverse Compton Emission, Astrophys. J. 520, 529-547 (1999)

27. Brunetti, G.; Blasi, P., Alfvenic reacceleration of relativistic particles in galaxy clusters in the presence of secondary electrons and positrons, Mon. Not. R. Astron. Soc. 363, $1173-1187(2005)$

28. Reimer, O.; Pohl, M.; Sreekumar, P.; Mattox, J.R., EGRET Upper Limits on the HighEnergy Gamma-Ray Emission of Galaxy Clusters, Astrophys. J. 588, 155-164 (2003)

29. Pfrommer, C \& Ensslin, T.A. Constraining the population of cosmic ray protons in cooling flow clusters with $\gamma$-ray and radio observations: Are radio mini-halos of hadronic origin ?, Astron. Astrophys. 413, 17-36 (2004)

30. Cassano, R.; Brunetti, G.; Setti, G., Statistics of giant radio haloes from electron reacceleration models, Mon. Not. R. Astron. Soc. 369, 1577-1595 (2006) 
Acknowledgements We acknowledge partial support from ASI-INAF I/088/06/0 and PRIN-INAF 2007. G.B. and R.C. acknowledge the CfA, NRL and NRAO for hospitality and partial support during the preparation of the manuscript. Basic research in radio astronomy at the Naval Research Laboratory is supported by 6.1 base funding. The National Radio Astronomy Observatory is operated by Associated University, Inc., under cooperative agreement with the National Science Foundation. We thank R. Athreya the staff of the GMRT for their helping during the observations. GMRT is run by the National Center for Radio Tata Institute of Fundamental Research.

Author Information Reprints and permissions information is available at npg.nature.com/reprintsandpermissions. Correspondence and requests for materials should be addressed to G. Brunetti (brunetti@ira.inaf.it).

Correspondence and requests for materials should be addressed to G.B. (email: brunetti@ira.inaf.it). 


\title{
A low-frequency radio halo associated with a cluster of galaxies
}

\section{Supplementary Information}

\author{
G. Brunetti ${ }^{1}$, S. Giacintucci ${ }^{1,2}$, R. Cassano $^{1}$, W. Lane $^{3}$, D. Dallacasa ${ }^{4}$, T. Venturi $^{1}$, \\ N. Kassim ${ }^{3}$, G. Setti ${ }^{1,4}$, W. D. Cotton ${ }^{5}, \&$ M. Markevitch ${ }^{2}$
}

To complement the information given in the main body of the Letter, these Sections provide supplementary discussion on the methods adopted to derive upper limits to the flux density of the halo reported in Figure 2 (Sect. 1) and on the origin of the halo (Sect. 2 and 3).

\section{Upper limits to the flux of the radio halo at $1.4 \mathrm{GHz}$}

The diffuse emission in the center of Abell 521 discovered by the low frequency radio observations presented in this Letter was not detected in VLA observations at $1.4 \mathrm{GHz}^{[17]}$. We re-analyzed those $\mathrm{BnC}$ VLA data taken from the archive. Given the sourthern declination of the cluster, they can be considered equivalent to a $\mathrm{C}$ configuration observation. The quality of the data is generally good, except for occasional intermittent RFI in IF2 $(1.44 \mathrm{GHz})$ and at the very end of the observation (both $25-\mathrm{MHz}$ IFs affected), when the source and its secondary calibrator were observed at low elevation and data on a few short baselines had corrupted amplitudes (very high circular polarization). RFI excision was carried

\footnotetext{
${ }^{1}$ INAF - Istituto di Radioastronomia, Via P. Gobetti 101, I-40129 Bologna, Italy

${ }^{2}$ Harvard-Smithsonian Center for Astrophysics, Cambridge, MA 02138, USA

${ }^{3}$ Naval Research Laboratory, Code 7213, Washington, DC 20375-5320, USA

${ }^{4}$ Dipartimento di Astronomia, Universita' di Bologna, Via Ranzani 1, I-40127 Bologna, Italy

${ }^{5}$ National Radio Astronomy Observatory, Charlottesville, Virginia 22903-2475, USA
}

out with great care. We also paid particular attention to the analysis of the secondary calibrator data, whose visibility turned out to be affected by substantial contribution from sources in the field. Therefore we first followed the standard calibration procedure, then we used the image of the secondary calibrator obtained in this way to improve the a-priori amplitude (and phase) calibration accuracy. The final dataset was obtained after a number of phase-only self-calibrations. Solutions had generally very high signal to noise ratio.

The final images were obtained by considering a central square, $0.8^{\circ}$ wide, with additional 10 smaller $\left(0.1^{\circ}\right)$ facets on confusing sources. In the full resolution image $\left(12.7 \operatorname{arcsec} \times 6.9 \operatorname{arcsec}\right.$ in p.a. $\left.75^{\circ}\right)$ the r.m.s. noise level is $15 \mu \mathrm{Jy} / \mathrm{beam}$. We also produced images with lower resolution by applying natural weighting to the data and also using a restricted uv-range.

A low resolution $30 \operatorname{arcsec} \times 30$ arcsec image of the region of Abell 521 is shown in Figure S1. Suspicious, very faint, diffuse emission appears in the region of the radio halo discovered at lower frequencies; however, most of this emission is due to the convolution of point sources in the same region (the image after subtraction of point sources is given in Figure 1) and the detection of the radio halo in Abell 521 at $1.4 \mathrm{GHz}$ would not have been firmly established from these observations only.

We thus estimated the upper limit to the flux density of the radio halo in these data at $1.4 \mathrm{GHz}$. This should account also for the possibility that a fraction of the flux density on the halo scale may be lost due 

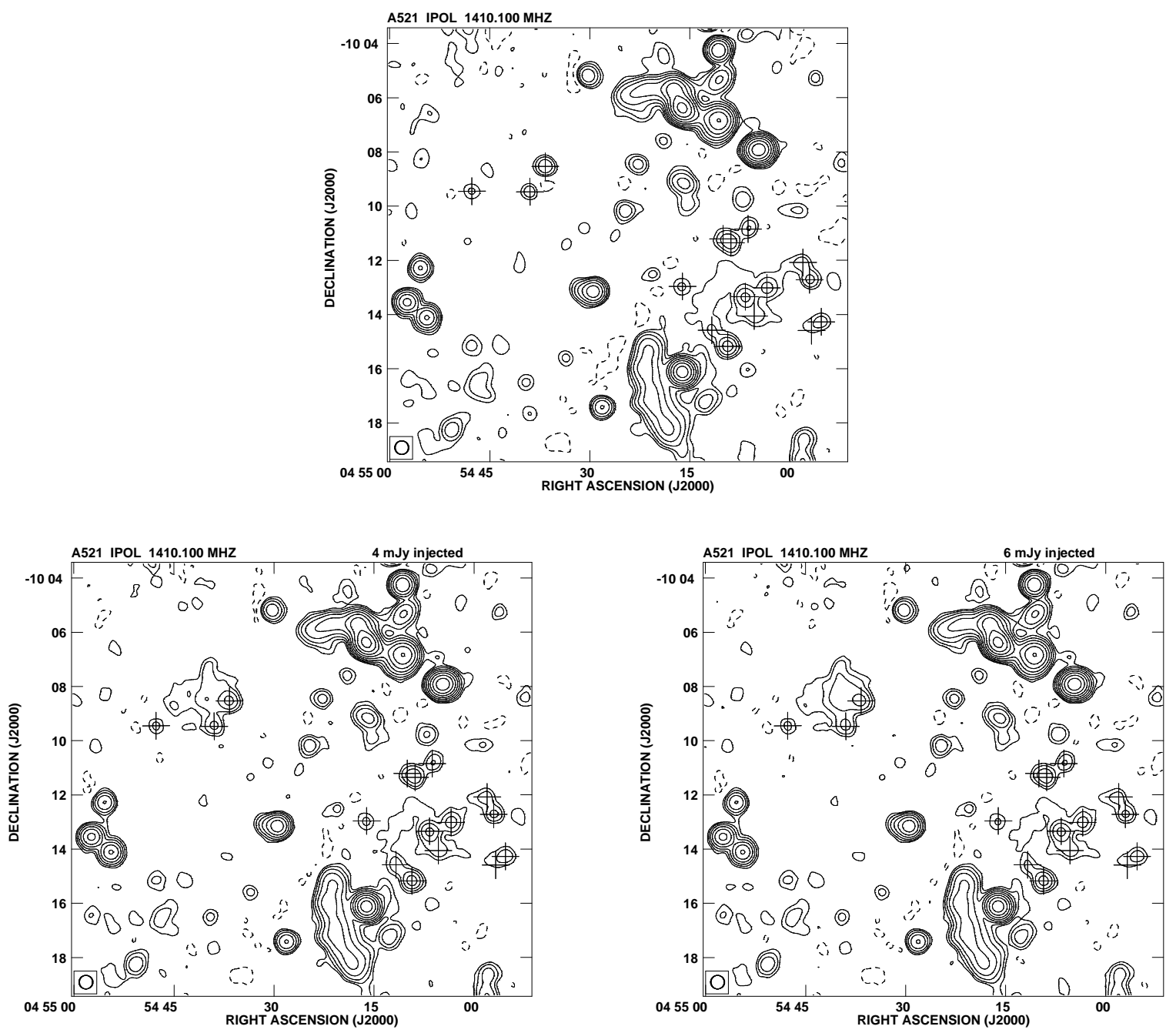

Figure 4: Low resolution images of Abell 521 at $1.4 \mathrm{GHz}$. The upper panel shows the image from our analysis of the original data, while lower panels show images from the analysis of the data containing injected fake radio halos with $\Theta_{50 \%}=2.15$ arcmin and $F=4$ (left) and 6 (right) mJy. Crosses mark the position of the point sources identified in the high resolution image in the central region of Abell 521 and in the region where fake radio halos have been injected. Radio contours are at $80,160,240, . . \mu \mathrm{Jy} / \mathrm{beam}$ level; negative contours at $-80 \mu \mathrm{Jy} / \mathrm{beam}$ level are reported in dashed lines. The r.m.s. noise level in the images is in the range $28-30 \mu \mathrm{Jy} / \mathrm{beam}$ and the restoring beam is $30 \operatorname{arcsec} \times 30 \operatorname{arcsec}$. 
to the uv coverage at short baselines of the observations. In order to derive a solid and conservative upper limit we follow ${ }^{[7,15]}$. We injected fake halos in the uv-components of the dataset by means of the AIPS task UVMOD ${ }^{[31]}$ and imaged the datasets with the same set of parameters applied to the original data. This procedure allows to evaluate the response of the $1.4 \mathrm{GHz}$ VLA observation to the presence of diffuse emission on the halo scale, as the uv components of the fake radio emission are sampled by means of the real uv coverage of the observation.

In particular, we model the brightness profile of the fake halos with sets of optically thin spheres with different radius and flux density, and obtain families of fake radio halos with total flux densities ranging from 3 to $15 \mathrm{mJy}$, with sizescale enclosing $50 \%$ of the flux $\Theta_{50 \%}=1.9-2.3$ arcmin $(\approx 440$ and $530 \mathrm{kpc}$, respectively) and maximum angular extension $\approx 5.2$ $\operatorname{arcmin}(\approx 1.2 \mathrm{Mpc})$.

Figure $\mathrm{S} 1$ shows the results obtained for fake radio halos with $\Theta_{50 \%}=2.15$ arcmin and injected flux density $F=4$ and $6 \mathrm{mJy}$. In the case of the halo with $F=4 \mathrm{mJy}$, about half of the injected flux is recovered by the analysis of the dataset, and the resulting diffuse emission in the image is still brighter than that at the center of Abell 521.

The analysis of the families of datasets with fake radio halos allowed us to derive a conservative upper limit to the flux density at $1.4 \mathrm{GHz}$ of the radio halo in Abell 521, $F<5$ mJy. This should be compared with the residual diffuse emission at $F \approx 1-2$ $\mathrm{mJy}$ level measured in the region of the radio halo after subtraction of discrete sources (Figure S1) in our images. Because the flux density of these sources, $F=3-4 \mathrm{mJy}$, is not negligible at $1.4 \mathrm{GHz}$, they were fitted in the full resolution image (with beam $=12.7 \operatorname{arcsec} \times 16.9 \operatorname{arcsec}$ and r.m.s. $=15 \mu \mathrm{Jy} / \mathrm{beam})$ by using the AIPS task JMFIT solving for a Gaussian component (which has then been subtracted out) and a non-zero level (i.e. diffuse emission, which is not subtracted).

\section{Projection effects and the radio halo}

Ghost radio plasma, confined within the intergalactic medium, may be revived by the passage of a shock wave through adiabatic compression ${ }^{32}$; indeed this mechanism has been proposed to explain radio relics as a scenario alternative to shock acceleration.

In principle, a large scale shock crossing Abell 521 along the line of sight may compress ghost radio plasma producing detectable synchrotron radiation with steep spectrum projected on the cluster central region, that may be classified as radio halo. However, the steep spectrum of the halo in Abell 521, its large $(\approx \mathrm{Mpc})$ extension and - in particular - the morphological correlation between radio halo and the Xray emission from the cluster can be used to conclude that this scenario is very unlikely.

In this context we have to assume that the bubble of ghost plasma extends on the Mpc scale. This implies a dynamical age of this plasma $\geq$ Gyr during which relativistic electrons lose energy via radiative and adiabatic losses. This results in a cutoff in the synchrotron spectrum emitted by the ghost plasma at very low frequencies, $v_{b, o} \leq \mathrm{MHz}$, before the shock-compression phase. The passage of the shock compresses the ghost plasma by a factor ${ }^{32}$ $x=\left(P_{d s} / P_{u s}\right)^{3 / 4}$, where $P_{d s}$ and $P_{u s}$ are the pressures measured in the thermal medium around the ghost plasma downstream and upstream the shock, respectively. The adiabatic compression boosts the frequency of the cut-off of the synchrotron spectrum at ${ }^{33} v_{c} \approx v_{c, o} x^{4 / 3} \approx P_{d s} / P_{u s}$. In order to have $v_{c} \approx 200-300 \mathrm{MHz}$ as measured in the spectrum of the radio halo in Abell 521, this would require $P_{d s} / P_{u s}>300$ implying a Mach number of the shock (from the relation between pressure jump and Mach number $\left.{ }^{34}\right)$ :

$$
M=\frac{1}{\sqrt{5}}\left(4 \frac{P_{d s}}{P_{u s}}+1\right)^{1 / 2}>15
$$

Strong large-scale shocks with $M>10$ are rare in galaxy clusters ${ }^{35,36,37}$ and may occur only outside the virialised region of the cluster where the temperature of the gas is well below $1 \mathrm{keV}$. Thus the compression of the ghost plasma should happen at $\geq 3$ Mpc distance from the center of Abell 521 (provided that a ghost-Gyr old plasma at such distances manages to survive from mixing with the intergalactic medium).

At the same time the radio halo is correlated with the cluster X-ray emission and its radio brightness decreases by a factor $\approx 6-8$ on the scale of the core 
of Abell 521. In order to reproduce this morphological connection by chance the ghost radio bubble, at a distance of $\geq 3 \mathrm{Mpc}$ from the cluster center, should be aligned with the center of Abell 521 within a projected distance of a cluster core-radius, $\approx 300 \mathrm{kpc}$. Geometrically, this implies that the direction connecting Abell 521 and the ghost bubble should make an angle $\theta \leq 7^{\circ}$ to the line of sight and the chance probability for that, $\approx 1-\cos (\theta)$, is $<1 \%$.

The ad hoc assumptions and this low probability allow us to conclude that this scenario is very unlikely.

\section{On the size of the radio relic in Abell 521}

The radio halo in Abell 521 appears connected with the radio relic (Figure 1). This is similar to that observed in the case of Abell $520^{33}$ and Abell 2256 38 ; a bridge connecting the halo and the relic is also observed in the Coma cluster ${ }^{39}$.

Radio relics are interpreted as the result of the acceleration of relativistic particles due to large scale shocks that form in the intergalactic medium during cluster mergers or accretion of matter ${ }^{40,41}$. The relic in Abell 521 is found to coincide with a possible shock front with Mach number $M \approx 2-4$, generated by the recent infall of a subcluster along the NorthWest/South-East direction ${ }^{[18]}$.

Electrons accelerated at the shock flow away into the intergalactic medium in a tail of emitting plasma, and one might expect that part of the emission in the region of the radio halo is due to this tail. However in the following we show that this is unplausible : the extension of the tail at a given frequency is constrained by the lifetime of the electrons emitting at that frequency (that depends on inverse Compton and synchrotron losses, and on adiabatic losses), which turns out to be much smaller than the $\geq \mathrm{Mpc}$ projected distance between the relic and halo region.

We adopt the most favorable geometry assuming that the shock is moving on the plane of the sky along the North-West/South-East direction, in which case the distance that separates the relic and halo region is not affected by projection effects. We also assume that expansion in the shocked gas plays a role on a timescale longer than the radiative timescale of the electrons emitting at $240 \mathrm{MHz}$, that is $\approx 0.2$
Gyr (Eq.1), and thus we neglect adiabatic losses of electrons. This last assumption is justified since the gas in the downstream region, over-pressured with respect to the ambient gas, should expand in a timescale that is a fraction of $\approx \phi_{r} / c_{s} \approx 0.5 \mathrm{Gyr}$, where $\phi_{r} \approx 500-700 \mathrm{kpc}$ is the longitudinal size of the relic ${ }^{18}$ and $c_{s}$ the sound velocity.

Under these assumptions, the size of the tail at a given frequency can be estimated as $l_{\text {tail }} \approx v_{d s} \tau_{r}$, where $\tau_{r}$ is the radiative lifetime of electrons emitting at that frequency, and $v_{d s}$ is the downstream velocity of the gas (in the shock frame, from the jump conditions $\left.{ }^{34}\right)$ :

$$
v_{d s}=c_{s}^{u} \frac{M^{2}+3}{4 M}
$$

where $c_{s}^{u}$ is the sound velocity in the intergalactic medium upstream of the shock. For a given Mach number of the shock $c_{s}^{u}$ can be estimated from the temperature of Abell 521 (taken as downstream temperature, and from the relations between temperature jump and Mach number ${ }^{34}$ ) :

$$
c_{s}^{u} \approx 1700 \sqrt{\frac{16 M^{2}}{\left(5 M^{2}-1\right)\left(M^{2}+3\right)}}(\mathrm{km} / \mathrm{s})
$$

Combining S.2-S.3 and adopting $\tau_{r} \approx 0.2 \mathrm{Gyr}$ for the electrons emitting at $240 \mathrm{MHz}$ (Eq. 1), we obtain $l_{\text {tail }} \approx 200 \mathrm{kpc}$ if the relic in Abell 521 in generated by a shock with $M \approx 2-4$.

Remarkably, $l_{\text {tail }} \approx 200 \mathrm{kpc}$ agrees with the transverse size of the relic at 240 and $330 \mathrm{MHz}$, and with the size of the synchrotron spectral steepening in the relic as measured from radio observations ${ }^{18}$.

\section{References}

1. AIPS: Astronomical Image Processing System, http://www.aips.nrao.edu/

2. Ensslin, T. A.; Gopal-Krishna, Reviving fossil radio plasma in clusters of galaxies by adiabatic compression in environmental shock waves, Astron. Astrophys. 366, 26-34 (2001)

3. Markevitch, M.; Govoni, F.; Brunetti, G.; Jerius, D., Bow Shock and Radio Halo in the Merg- 
ing Cluster A520, Astrophys. J. 627, 733-738 (2005)

4. Landau, L. D.; Lifshitz, E. M., Fluid Mechanics, (Pergamon Press) (1966)

5. Gabici, S.; Blasi, P., Nonthermal Radiation from Clusters of Galaxies: The Role of Merger Shocks in Particle Acceleration, Astrophys. J. 583, 695-705 (2003)

6. Pfrommer, C.; Ensslin T. A.; Springel, V.; Jubelgas, M.; Dolag, K., Simulating cosmic rays in clusters of galaxies - I. Effects on the SunyaevZel'dovich effect and the X-ray emission, Mon. Not. R. Astron. Soc. 378, 385-408 (2007)

7. Vazza, F.; Brunetti, G., Gheller, C.; Shock Waves in Eulerian Cosmological Simulations: Main Properties and Acceleration of Cosmic Rays, Mon. Not. R. Astron. Soc., submitted, astro-ph/0808.0609

8. Clarke, T. E.; Ensslin, T. A., Deep $1.4 \mathrm{GHz}$ Very Large Array Observations of the Radio Halo and Relic in Abell 2256, Astron. J. 131, 2900-2912 (2006)

9. Venturi, T.; Giovannini, G.; Feretti, L., Highsensitivity radio observations of the Coma cluster of galaxies, Astron. J. 99, 1381-1396 (1990)

10. Ensslin, T. A.; Biermann, P. L.; Klein, U.; Kohle, S., Cluster radio relics as a tracer of shock waves of the large-scale structure formation, Astron. Astrophys. 332, 395-409 (1998)

11. Roettiger, K.; Burns, J. O.; Stone, J. M., A Cluster Merger and the Origin of the Extended Radio Emission in Abell 3667, Astrophys. J. 518, 603612 (1999) 